\title{
MULTIPLE OBJECTIVE OPTIMIZATION OF SUBMERGED ARC WELDING PROCESS PARAMETERS USING GREY BASED FUZZY LOGIC
}

\author{
Edwin Raja Dhas, J. ${ }^{*}$ Satheesh, M.. ${ }^{* *}$ \\ *Department of Automobile Engineering, \\ ${ }^{* *}$ Department of Mechanical Engineering, \\ Noorul Islam Centre for Higher Education, Kumaracoil, India \\ E-mail: edwinrajadhas@rediffmail.com
}

\begin{abstract}
:
In the present work, an attempt has been made to apply an efficient technique, Grey based fuzzy logic method to solve correlated multiple response optimization problems, in the field of submerged arc welding. This approach converts the complex multiple objectives into a single grey-fuzzy reasoning grade. Based on grey-fuzzy reasoning grade, optimum levels of parameters (Welding current, arc voltage and welding speed) are identified. Nine experiments based on an orthogonal array of Taguchi method were performed. Weld bead hardness and material deposition rate were selected as the quality targets. The optimal procedure is proposed and developed for solving the optimal multi-response problem, which applies the grey relational coefficient in each response and converts a grey-fuzzy reasoning grade so as to evaluate multiple responses. The significant contributions of parameters are estimated using Analysis Of Variance (ANOVA). Confirmation test is conducted and reported. It is found that the welding current is the most significant controlled factor for the process according to the weighted sum grade of the maximum weld bead hardness and material deposition rate. This evaluation procedure can be used in intelligent decisionmaking for a welding operator. The proposed and developed method has good accuracy and competency. The paper highlights a detailed methodology of the proposed scheme and its effectiveness. The proposed technique provides manufacturers to develop intelligent manufacturing system to achieve the highest level of automation.
\end{abstract}

Key Words: Parameter Optimization, Orthogonal Array, Grey Based Fuzzy Logic, ANOVA, Submerged Arc Weld, Process Parameters

\section{INTRODUCTION}

Welding is one of the major and principal manufacturing processes widely used because of its inherent properties of joining similar or dissimilar metals efficiently and economically. It is a multi-input multi-output process since the output variables being closely coupled together, a great deal of time and cost are expended by trial-and-error methods to obtain optimal weld conditions through the combination of the various welding process parameters to produce consistent weld quality. SAW process is a widely used industrial arc welding process needs a better prediction and monitoring of its parameters. Process planners use different techniques to estimate the influence of the welding parameters (welding current, arc voltage, and welding speed) on bead hardness and deposition rate which are the most sought weld quality indicator in the SAW process. Weld is sound and economical if it achieves maximum deposition rate and hardness [1-3]. Usually, the desired welding parameters are determined based on welder's experiences which are simple and inexpensive. However, the obtained result may not guarantee optimal performance. It is necessary to select the most appropriate weld parameter settings to improve weld efficiency, process at low cost and produce highquality products. 
Weld parameters optimization through experimental methods and mathematical models has grown substantially over time to achieve a common goal of improving higher weld efficiency [4-6]. The applications of Taguchi's method optimize the performance characteristics through the settings of process parameters and reduce the sensitivity of the system performance to sources of variation and have become a powerful design of experiments method $[7,8]$. Traditional Taguchi method can solve single and simple objective problems of. But in the real world engineering applications most of the problems are multi-objective [9]. It is cumbersome to optimize simultaneously objectives in complex process by single objective method and engineering judgment is primarily used to resolve such complicated problems. An engineering judgment often increases the degree of uncertainty during efficient decisionmaking process. Hence solving multiple objectives problem needs attention.

Grey relational analysis theory initialized by Deng [10] makes use of this technique to handle uncertain systematic problem with only partial known information. Grey relational analysis theory is adopted for solving the complicated interrelationships among the multiple objectives in various fields of manufacturing [11-17].

The theory of fuzzy logic originated by Zadeh [18] has been proven to be useful for dealing with uncertain and vague information. The definition of objectives contains certain degree of uncertainty with vagueness. Hence the fuzzy logic is applied to establish the optimal setting of parameters for multiple objectives. This method is applied to optimize the parameters of electrical discharge machining [19, 20].

The grey based fuzzy logic approach combines the advantage of both the methods. The objectives in grey method such as lower-the-better, higher-the-better and nominal-the-better contains certain degree of uncertainty and vagueness which is overcome by fuzzy logic. This method was successfully applied to optimize multiple objectives of complicated problems in machining parameter optimization [21], optimize parameters in light guide plate printing process [22] and optimize parameters in CNC turning process [23]. Fuzzy based desirability function approach [24] was used to optimize of bead geometry of submerged arc weld. In this study, the effect of weld parameters on bead hardness and deposition rate are reported using grey based fuzzy logic. The significant contribution of each cutting parameters to the multiple objectives are calculated using ANOVA [25] analysis.

\section{GREY BASED FUZZY LOGIC}

The proposed approach combines the grey relational analysis with fuzzy logic in order to determine the process parameters with optimal performance characteristics.

\subsection{Grey Relational Analysis}

Grey relational analysis is used to solve complicated interrelationships among the multiple performance characteristics problems effectively. In grey relational analysis, system has a level of information between black and white. In other words, in a grey system, some information is known and some information is unknown. In a white system, the relationships among factors in the system are certain; in a grey system, the relationships among factors in the system are uncertain.

Data pre-processing is normally required since the range and unit in one data sequence may differ from the others. Data preprocessing is also necessary when the sequence scatter range is too large, or when the directions of the target in the sequences are different. Data pre-processing is a means of transferring the original sequence to a comparable sequence. Depending on the characteristics of a data sequence, there are various methodologies of data pre-processing available for the grey relational analysis. Experimental data $y_{i j}$ is normalized as $Z i j(0 \leq Z i j \leq 1)$ for the $i^{\text {th }}$ performance characteristics in the $j^{\text {th }}$ experiment can be expressed as: 
For Larger-the-better condition

$$
Z_{i j}=\frac{y_{i j}-\min \left(y_{i j,}, i=1,2, \ldots . n\right)}{\max \left(y_{i j}, i=1,2, \ldots . n\right)-\min \left(y_{i j}, i=1,2, \ldots \ldots n\right)}
$$

For smaller-the-better

$$
Z i j=\frac{\max \left(y_{i j,}, i=1,2, \ldots . . n\right)-y_{i j}}{\max \left(y_{i j}, i=1,2, \ldots \ldots n\right)-\min \left(y_{i j}, i=1,2, \ldots . . n\right)}
$$

For nominal-the-best

$$
Z_{i j}=\frac{\left(y_{i j}-\text { Target }\right)-\min \left(\left|y_{i j}-\operatorname{Target}\right|, i=1,2, \ldots \ldots n\right)}{\max \left(\mid y_{i j}-\text { Target } \mid, i=1,2, \ldots . . n\right)-\min \left(\mid y_{i j}-\text { Target } \mid, i=1,2, \ldots \ldots n\right)}
$$

Then, calculate grey relational co-efficient ${ }^{\gamma_{i j}}$ for the normalized values.

$$
\gamma_{i j}=\frac{\Delta \min +\xi \Delta \max }{\Delta o j(k)+\xi \Delta \max }
$$

Where
a) $\quad j=1,2 . . n ; k=1,2 . . m, n$ is the number of experimental data items and $m$ is the number of responses.
b) $\quad y_{0}(k)$ is the reference sequence $\left(y_{0}(k)=1, k=1,2 . . m\right) ; y_{j}(k)$ is the specific comparison sequence.
c) $\Delta_{o j}=\left\|y_{o}(k)-y_{j}(k)\right\|_{=}$The absolute value of the difference between $y_{0}(k)$ and $y_{j}(k)$
d) $\Delta \min =\min _{\forall j \in i} \min _{\forall k}\left\|y_{o}(k)-y_{j}(k)\right\|$ is the smallest value of $y_{j}(k)$
e) $\Delta \max =\max _{\forall j \in i} \max _{\forall k}\left\|y_{o}(k)-y_{j}(k)\right\|$ is the largest value of $y_{j}(k)$
f) $\quad \zeta$ is the distinguishing coefficient which is defined in the range $0 \leq \zeta \leq 1$ (the value may adjusted based on the practical needs of the system)

This grey relation co-efficient is applied to show the relationship between the optimal (best=1) and actual normalized results. The higher value of grey relational coefficient $\gamma_{i j}$ represents that the corresponding experimental results is closer to the optimal (best) normalized value for the single response.

\subsection{Fuzzy Logic}

Fuzzy logic (Figure 1) is a way to representing information that mimics human reasoning about information. The most interesting fact about fuzzy logic is that fuzzy inferences make it possible to deduce a proposition similar to the consequence from some proposition that is similar to the antecedent [26, 27]. It is an effective mathematical model of resolving problems in a simple way which contain the uncertain and huge information [28]. In fuzzy logic analysis, the fuzzifier uses membership functions to fuzzify the grey relational coefficient. The fuzzy inference engine then performs a fuzzy inference on fuzzy rules in order to 
generate a fuzzy value. Finally, the defuzzifier converts the fuzzy value into a grey-fuzzy reasoning grade. Fuzzy logic has three simple steps defined below

Step 1 Fuzzification: In a fuzzy expert system application, each input variable's crisp value is first fuzzified into linguistic values before the inference engine proceeds in processing with the rule base.

Step 2 Inference engine: In a fuzzy expert system, the collection of fuzzy IF-THEN rules is stored in the fuzzy rule base which is referred to by the inference engine when processing inputs. Once all crisp input values have been fuzzified into their respective linguistic values, the inference engine will access the fuzzy rule base of the fuzzy expert system to derive linguistic values for the intermediate as well as the output linguistic variables. The grey relational coefficients $x_{1}, x_{2}, x_{n}$ and a multi-objective output $y$ that is of the form

Rule 1: IF $x_{1}$ is $A_{11}$ and $x_{2}$ is $A_{21} \ldots$ and $x_{n}$ is $A_{n 1}$ THEN $y$ is $c_{1}$ else

Rule 2: IF $x_{1}$ is $A_{12}$ and $x_{2}$ is $A_{22} \ldots$ and $x_{n}$ is $A_{n 2}$ THEN $y$ is $c_{2}$ else

$\vdots$

Rule $i$ : IF $x_{1}$ is $A_{1 i}$ and $x_{2}$ is $A_{2 i} \ldots$ and $x_{n}$ is $A_{n i}$ THEN $y_{\text {is }} c_{i}$ else

$\vdots$

Rule $n$ : IF $x_{1 \text { is }} A_{1 n}$ and $x_{2}$ is $A_{2 n} \ldots$ and $c_{i}$ is $A_{n n}$ THEN $y_{\text {is }} c_{n}$ else

where $A_{1 i}, A_{2 i}, \ldots, A_{n i}$ and $C_{i}$ are fuzzy subsets defined by the corresponding MFs, i.e., $\mu$ $A_{1 i}, \mu A_{2 i}, \ldots, A_{n i}$ and $\mu c_{i}$. The fuzzy multi-objective output $y$ is provided from those above rules by employing the max-min interfaced operation. Inference results in a fuzzy set with MF for the multi-response output ${ }^{y}$ can be expressed as following

$\mu C_{0}(y)=\left(\mu A_{11}\left(x_{1}\right) \wedge \mu A_{21}\left(x_{2}\right) \ldots \mu C_{1}(y)\right) \ldots \vee\left(\mu A_{1 n}\left(x_{1}\right) \wedge \mu A_{2 n}\left(x_{2}\right) \ldots \mu C_{n}(y)\right)$

Where $\wedge$ and $\vee$ are the minimum operation and maximum operation.

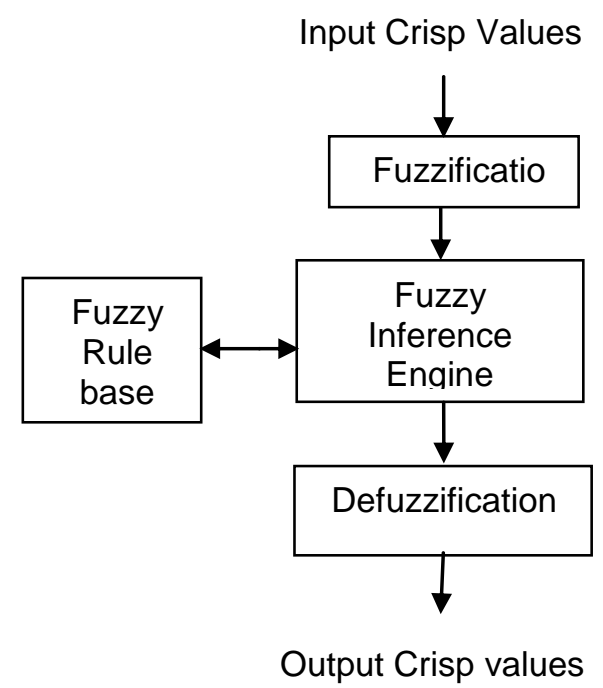

Figure 1: Stucture of a Fuzzy Expert System Model.

Step 3 Defuzzification: The last phase in the fuzzy expert system is the defuzzification of the linguistic values of the output linguistic variables into crisp values. The most common techniques for defuzzification are Center-of-Maximum (CoM) and Center-of-Area (CoA). The 
fuzzy inference output $\mu C_{0}(y)$ transferred to a non-fuzzy value $y_{0}$ using the centroid defuzzification method, i.e.

$$
y_{0}=\frac{\sum y \mu C_{0}(y)}{\sum \mu C_{0}(y)}
$$

This non-fuzzy value $y_{0}$ is called as grey-fuzzy reasoning grade. The grey-fuzzy reasoning grade is used to optimize the multiple objectives and the relational degree between main factor and other factors for each performance characteristics. The higher grey-fuzzy reasoning grade indicates that the experimental result closer to the ideally normalized value.

\section{PROPOSED METHODOLOGY}

The scheme of the proposed grey based fuzzy logic approach is (Fig.2) summarized as follows

Step 1: Design an appropriate orthogonal array to plan the experimental design and determining the level of parameters

Step 2: Conduct the experiments based on the orthogonal array

Step 3: Data pre-processing of experimental results using (1) - (3)

Step 4: Compute the grey relational coefficient of machining objectives by using (4)

Step 5: Establishing the trapezoidal membership function and fuzzy rule to fuzzify the grey relational coefficient of each response.

Step 6: Calculating the fuzzy multi-objectives output by defuzzification of the output linguistic variables into crisp values i.e., grey-fuzzy reasoning grade.

Step 7: Mean response for each level of parameters is calculated and their response graph is plotted.

Step 8: Select the optimal level setting of machining parameters by using response table and response graph.

Step 9: Analyze the grey-fuzzy reasoning grade with ANOVA.

Step10: Verify the optimal process parameters through Confirmation test.

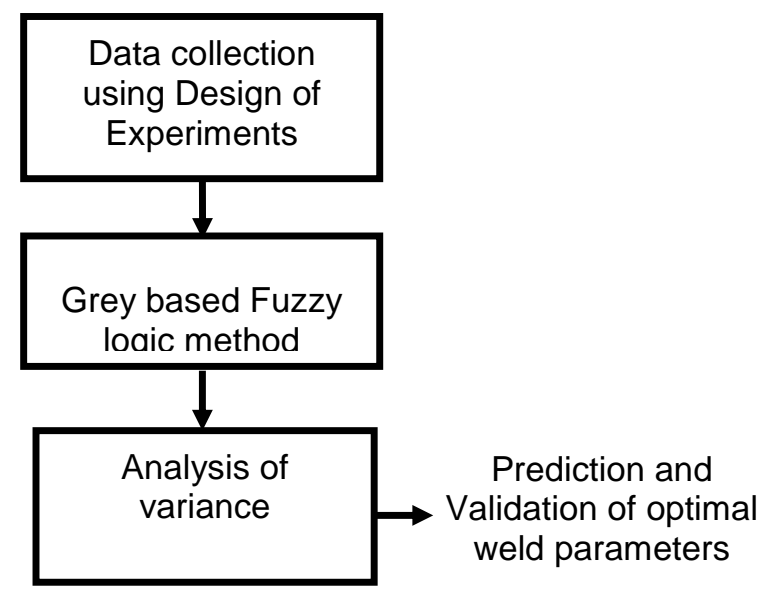

Figure 2: Scheme of the proposed grey based fuzzy logic method.

\subsection{Determination of optimal weld parameters}

Experiments are designed as per Taguchi's $L_{9}$ orthogonal array layout to avoid systematic errors penetrating the system at $\mathrm{M} / \mathrm{s}$. Sree Jyothi Enterprises, Tiruchirappali, India. TORNADO SAW M-800 semiautomatic welding equipment with a constant voltage, rectifier type power source, 800 A capacity was used to join two mild steel plates of dimension $200 \mathrm{X}$ 100 X 6 mm, IS: 2062 (0.25 \% C, $0.2 \%$ Si, $0.75 \%$ Mn and balance Fe). Copper coated 
electrodes of AWS EL8CCMS, $4 \mathrm{~mm}$ diameter in coil form and basic- fluoride- type (equivalent to DIN 8557) granular flux were used (Fig.3). Square butt joint with a $1 \mathrm{~mm}$ root opening was selected to join the plates in the flat position, keeping the electrode positive and perpendicular to the plate as per the experimental setup explained previously. Single pass welding bead on joint weld with square butt weld is performed on the weld plates by varying the initial parameters as shown in Table I. The working ranges for the process parameters were selected from American Society Welding handbook. Each trial of experiment was done twice and the average value is taken.

Deposition rate and bead hardness are considered as objectives. The metal deposition rate was calculated with the help of stop watch and length of the electrode melt. Vickers hardness test was performed on Vickers Hardness testing machine having diamond indenter by applying a load of $40 \mathrm{~kg}$. Based on the designed $\mathrm{L}_{9}$ orthogonal array combination a series of joining processes are performed in welding machine. Experimental results are summarized in Table II.

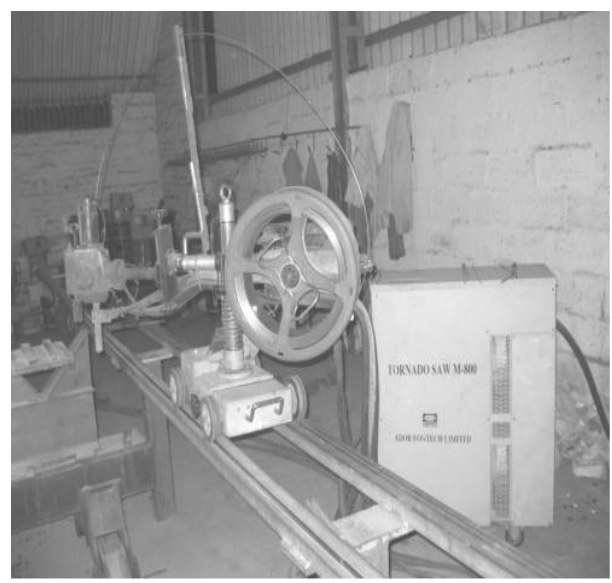

Figure 3: Schematic diagram of experimental set-up.

Table I: Welding parameters and their levels.

\begin{tabular}{|c|l|c|c|c|}
\hline Factors & Welding Parameters & Level 1 & Level 2 & Level 3 \\
\hline A & Welding Current, A & 305 & 310 & 315 \\
B & Arc Voltage, V & 25 & 26 & 27 \\
C & Welding Speed, cm/min & 25 & 26 & 27 \\
\hline
\end{tabular}

Table II: Experimental results from experimentation.

\begin{tabular}{|c|c|c|c|c|c|}
\hline $\begin{array}{c}\text { Trial } \\
\text { Nos }\end{array}$ & A & B & C & $\begin{array}{c}\text { Deposition rate, } \\
\mathrm{Mm}^{3} / \mathrm{s}\end{array}$ & $\begin{array}{c}\text { Hardness, } \\
\text { HV }\end{array}$ \\
\hline 1 & 1 & 1 & 1 & 173.7 & 630.6122 \\
\hline 2 & 1 & 2 & 2 & 161.56 & 649.0233 \\
\hline 3 & 1 & 3 & 3 & 170.40 & 647.2387 \\
\hline 4 & 2 & 1 & 2 & 185.17 & 630.6122 \\
\hline 5 & 2 & 2 & 3 & 160.38 & 674.8526 \\
\hline 6 & 2 & 3 & 1 & 164.25 & 607.2583 \\
\hline 7 & 3 & 1 & 3 & 160.77 & 688.3493 \\
\hline 8 & 3 & 2 & 1 & 183.52 & 617.4078 \\
\hline 9 & 3 & 3 & 2 & 175.99 & 610.3103 \\
\hline
\end{tabular}




\subsection{Optimization of welding parameters}

Normalize the value of given objectives using one of the Equations (1), (2) and (3) depending upon the type of quality characteristics. Calculate the grey relational co-efficient using (4). The value for $\xi$ is taken as 0.5 in (4) since all the process parameters are of equal weighting. The computed normalized value for each quality characteristics and grey relational coefficients are shown in Table III.

Table III: Normalized value and Grey relational coefficients of responses.

\begin{tabular}{|c|c|c|c|c|}
\hline $\begin{array}{c}\text { Exp. } \\
\text { No. }\end{array}$ & \multicolumn{2}{|c|}{ Normalized values of } & \multicolumn{2}{c|}{$\begin{array}{c}\text { Grey Relational } \\
\text { Coefficients of }\end{array}$} \\
\cline { 2 - 5 } & $\begin{array}{c}\text { Deposition } \\
\text { rate }\end{array}$ & $\begin{array}{c}\text { Vickers } \\
\text { Hardness }\end{array}$ & Deposition rate & $\begin{array}{c}\text { Vickers } \\
\text { Hardness }\end{array}$ \\
\hline 1 & 0.53731 & 0.28799 & 0.51938 & 0.41254 \\
\hline 2 & 0.0476 & 0.51503 & 0.344258 & 0.507634 \\
\hline 3 & 0.40419 & 0.49303 & 0.456286 & 0.49654 \\
\hline 4 & 1 & 0.28799 & 1 & 0.41254 \\
\hline 5 & 0 & 0.83356 & 0.333333 & 0.750256 \\
\hline 6 & 0.15611 & 0 & 0.372055 & 0.333333 \\
\hline 7 & 0.01573 & 1 & 0.336866 & 1 \\
\hline 8 & 0.93344 & 0.12516 & 0.88252 & 0.363679 \\
\hline 9 & 0.62968 & 0.03763 & 0.574508 & 0.341912 \\
\hline
\end{tabular}

Next, the grey relational grade is computed using fuzzy logic analysis. Grey relational coefficients of bead hardness and deposition rate are taken as input to the fuzzy model and the output is grey-fuzzy reasoning grade. MATLAB software is employed to develop the fuzzy logic model (Figure 4). The grey relational coefficients corresponding to each process response is fuzzified and then a single fuzzy reasoning grade is obtained by using the maxmin fuzzy inference and centroid defuzzification methods. Hence, optimization of complicated multiple process responses is converted into optimisation of a single fuzzy reasoning grade. The defuzzifier converts the fuzzy value into non-fuzzy value which is called as grey-fuzzy reasoning grade. The membership function adopts in this is trapezoidal membership function which has a flat top and really is just a truncated triangle curve (Figure 4). There are five fuzzy sets for variables bead hardness and deposition rate: (Figure 5) very small (VS), small $(\mathrm{S})$, medium $(\mathrm{M})$, large (L), and very large (VL). The nine fuzzy sets (Figure 6) are: tiny (T), very small (VS), small (S), small medium (SM), medium (M), medium large (ML), large (L), very large $(\mathrm{VL})$, and huge $(\mathrm{H})$. The fuzzy rules in a matrix form used for the fuzzy logic controller are presented in Table IV.

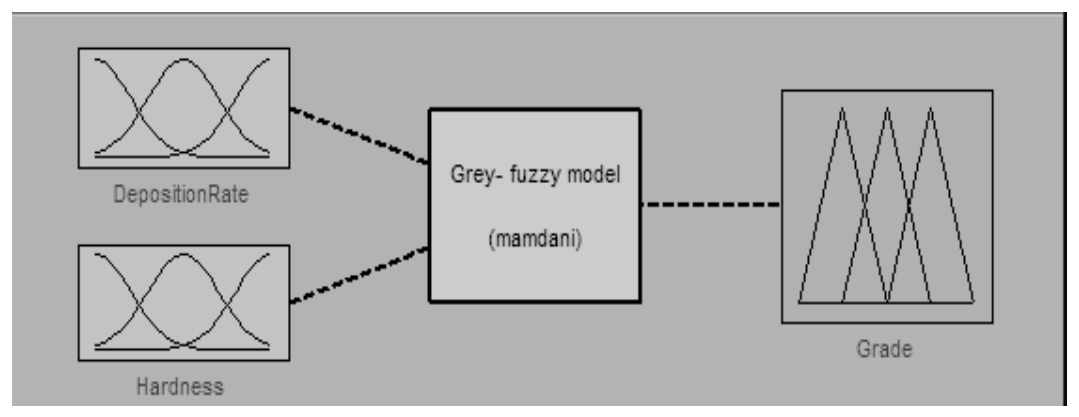

Figure 4: Fuzzy logic model for welding responses. 


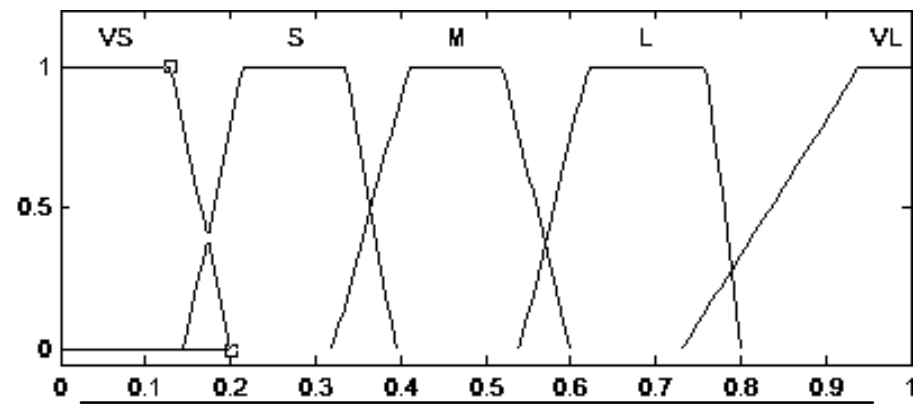

Figure 5: Membership function for deposition rate and hardness.

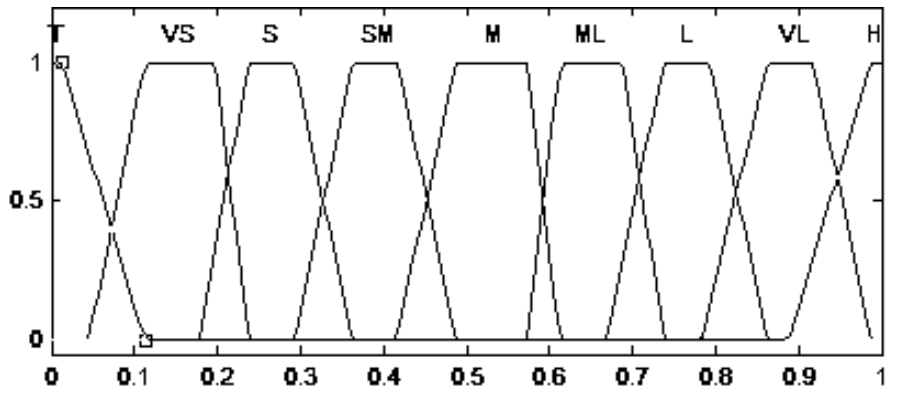

Figure 6: Membership function for grey-fuzzy reasoning grade.

Table IV: Fuzzy rules in matrix form.

\begin{tabular}{|c|c|c|c|c|c|c|}
\hline \multirow{2}{*}{\multicolumn{2}{|c|}{$\begin{array}{l}\text { Fuzzy rule for grey- } \\
\text { fuzzy model }\end{array}$}} & \multicolumn{5}{|c|}{ Normalized value of bead hardness } \\
\hline & & VS & $\mathrm{S}$ & $\mathrm{M}$ & $\mathrm{L}$ & $\overline{V L}$ \\
\hline \multirow{5}{*}{$\begin{array}{l}\text { Normalized } \\
\text { Value of } \\
\text { deposition } \\
\text { rate }\end{array}$} & VS & $\mathrm{T}$ & VS & $S$ & SM & $\mathrm{M}$ \\
\hline & $\mathrm{S}$ & VS & $\mathrm{S}$ & SM & $M$ & $\overline{M L}$ \\
\hline & $\mathrm{M}$ & S & $\overline{S M}$ & $\mathrm{M}$ & $\mathrm{ML}$ & $L$ \\
\hline & $\mathrm{L}$ & SM & $\mathrm{M}$ & $\mathrm{ML}$ & $\mathrm{L}$ & $\mathrm{VL}$ \\
\hline & $\overline{V L}$ & $M$ & $\mathrm{ML}$ & $\mathrm{L}$ & VL & $\mathrm{H}$ \\
\hline
\end{tabular}

Graphical representation of fuzzy logic reasoning procedure for the experimental result is shown (Figure 7). The rows represent 25 rules, and columns represent the two inputs and one output variable. The locations of trapezoidal indicates the determined fuzzy sets for each input and output value. The height of the darkened area in each trapezoidal corresponds to the fuzzy membership value for that fuzzy set. For the first experiment, the input value of grey relational coefficient of deposition rate and hardness are 0.519 and 0.412 respectively. The defuzzified output for the first experiment gives the grey-fuzzy reasoning grade value as 0.479 from the combined darkened areas shown in the last column of Grey-fuzzy-grade (Figure 7). The entire results of the calculated grey-fuzzy reasoning grade for the experiments are displayed in Table V. Finally, the grades are considered for optimizing the multi objective parameter design problem. 


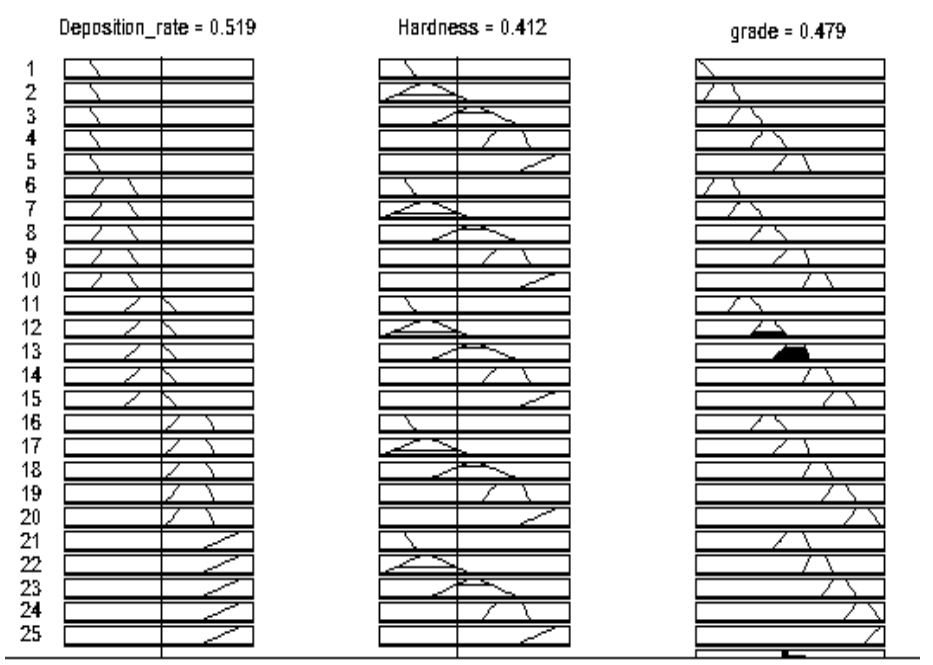

Figure 7: Fuzzy logic rules viewer for the model Exp 1.

From the value of grey-fuzzy reasoning grade (Table V), the main effects are tabulated in Table VI and the factor effects are plotted (Figure 7). Considering maximization of grade value (Table VI / Figure 8), optimal parameter conditions $\mathrm{A}_{3} \mathrm{~B}_{1} \mathrm{C}_{3}$ are obtained.

Table V: Grey-fuzzy reasoning grade and their rank.

\begin{tabular}{|c|c|c|}
\hline Exp. No. & GR Grade & Rank \\
\hline 1 & 0.479 & 7 \\
\hline 2 & 0.425 & 8 \\
\hline 3 & 0.521 & 5 \\
\hline 4 & 0.733 & 1 \\
\hline 5 & 0.556 & 4 \\
\hline 6 & 0.386 & 9 \\
\hline 7 & 0.675 & 3 \\
\hline 8 & 0.708 & 2 \\
\hline 9 & 0.519 & 6 \\
\hline
\end{tabular}

Table VI: Response table for the grey-fuzzy reasoning grade.

\begin{tabular}{|c|c|c|c|}
\hline Factors & Level-1 & Level-2 & Level-3 \\
\hline A & 0.475 & 0.558 & 0.634 \\
\hline B & 0.629 & 0.563 & 0.475 \\
\hline C & 0.524 & 0.559 & 0.584 \\
\hline
\end{tabular}

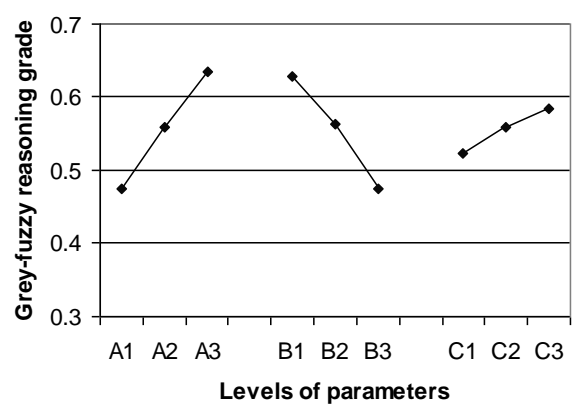

Figure 8: The response graph for each level of welding parameters. 


\section{RESULTS}

Using the grey relational grade value, ANOVA is formulated for identifying the significant factors. The results of ANOVA are given in Table VII. From ANOVA, it is clear that welding current $(30.76 \%)$ influences more on welding of mild steel followed by welding voltage $(28.94 \%)$ and welding speed (4.44\%).

Table VII: ANOVA results of grey-fuzzy reasoning grade values.

\begin{tabular}{|c|c|l|l|c|c|}
\hline Factor & $\begin{array}{c}\text { Degrees } \\
\text { of } \\
\text { freedom }\end{array}$ & $\begin{array}{l}\text { Sum of } \\
\text { Square }\end{array}$ & Mean Square & F value & $\begin{array}{c}\% \\
\text { Contribution }\end{array}$ \\
\hline A & 2 & 0.0379 & 0.01898 & 0.86 & 30.76 \\
\hline B & 2 & 0.03565 & 0.01783 & 0.81 & 28.94 \\
\hline C & 2 & 0.00539 & 0.00269 & 0.12 & 4.44 \\
\hline Error & 2 & 0.04418 & 0.02209 & - & 35.86 \\
\hline Total & 8 & 0.12318 & - & - & 100 \\
\hline
\end{tabular}

In order to predict the optimum condition, the expected mean at the optimal settings $(\mu)$ is calculated by using the following model.

$$
\mu=\bar{A}_{3}+\bar{B}_{1}+\bar{C}_{3}-2 \times \bar{T}_{\mathscr{B}}
$$

Where, $\bar{A}_{3}, \bar{B}_{1}$ and $\bar{C}_{3}$ and are the mean values of the grey relational grade with the parameters at optimum levels and $\bar{T}_{g g}$ is the overall mean of average grey grade. The expected mean $(\mu)$ at optimal setting is found to be 0.736 .

Confidence interval $(\mathrm{Cl})$ is calculated as

$$
\begin{aligned}
C l & =\sqrt{F_{\alpha}\left(1, f_{e}\right) V_{e}\left[\frac{1}{n_{\text {eff }}}+\frac{1}{R}\right]} \\
& = \pm 0.3172
\end{aligned}
$$

Where, $F_{\alpha}\left(1, f_{e}\right)$ is the $\mathrm{F}$ ratio at a significance level of $\alpha \%, \alpha$ is the risk, $f_{e}$ is the error degrees of freedom, $V_{e}$ is the error mean square, $n_{\text {eff }}$ is the effective total number of tests and $R$ is the number of confirmation tests

$$
n_{\text {eff }}=\frac{\text { Total number of observations }}{1+\text { Total degrees of freedom associated with items used in estimating } \mu}
$$

Therefore $95 \%$ confidence interval of the predicted optimum condition is given by following model, where $\mu=$ the Grey relational grade value after conducting the confirmation experiments with optimal setting point, i.e., $A_{2} B_{2} C_{3}$

$$
\begin{gathered}
(0.712-0.3172)<\mu<(0.712+0.3172) \\
(0.3948)<\mu<(1.0292)
\end{gathered}
$$


Once the optimal level of the design parameters has been determined, the final step is to predict and verify the quality characteristic using this optimal level of design parameters. Table VIII gives a comparison of the multiple process responses for initial and optimal welding parameters. As noted from Table VIII, the deposition rate is increased from 160.38 $\mathrm{mm}^{3} / \mathrm{s}$ to $161.12 \mathrm{~mm}^{3} / \mathrm{s}$ and the hardness is increased from $674.85 \mathrm{HV}$ to $689.21 \mathrm{HV}$ respectively. It is clearly shown that the multiple performance characteristics in the welding process are together improved.

Table VIII: Results of initial and optimal weld performance.

\begin{tabular}{|c|c|c|}
\hline \multirow{2}{*}{$\begin{array}{c}\text { Initial Welding } \\
\text { parameters }\end{array}$} & \multicolumn{2}{|c|}{ Optimal welding parameters } \\
\cline { 2 - 3 } & Prediction & Experiment \\
\hline Levels $\mathrm{A}_{2} \mathrm{~B}_{2} \mathrm{C}_{3}$ & $\mathrm{~A}_{3} \mathrm{~B}_{1} \mathrm{C}_{3}$ & $\mathrm{~A}_{3} \mathrm{~B}_{1} \mathrm{C}_{3}$ \\
\hline Deposition rate 160.38 & 160.77 & 161.12 \\
\hline Hardness 674.85 & 688.34 & 689.21 \\
\hline $\begin{array}{c}\text { Grey relational grade } \\
0.556\end{array}$ & 0.736 & 0.712 \\
\hline $\begin{array}{c}\text { Improvement of grey } \\
\text { relational grade }\end{array}$ & 0.180 & 0.156 \\
\hline
\end{tabular}

\section{CONCLUSIONS}

The effects of welding parameters and the optimum welding parameters for a SAW process on the multiple performance characteristics are systematically investigated by grey relational analysis and fuzzy logic with orthogonal array. The following conclusions are made:

- Approach of grey based fuzzy logic analysis is a productive method for optimizing the multi-objective problems predicting the deposition rate and hardness in welding of mild steel.

- From ANOVA computations, it is revealed that welding current and welding voltage are predominant factors which affect the welding of mild steel. Welding current $(30.76 \%)$ influences more on welding of mild steel followed by arc voltage $(28.94 \%)$ and welding speed $(4.44 \%)$.

- The best performance characteristics is obtained with welding current of $315 \mathrm{~A}$, lower arc voltage of $25 \mathrm{~V}$ and higher welding speed of $27 \mathrm{~cm} / \mathrm{s}$.

- Through the optimum procedure of grey-fuzzy logic with orthogonal array, the level constitution of optimal welding parameters are acquired and verified by confirmation test. Final results verifies that the multiple objectives are improved simultaneously through this approach.

\section{REFERENCES}

[1] Howard Cary, B. (1979). Modern Welding Technology, Prentice Hall Inc, Englewood Cliffs, NJ

[2] Nadkarni, S.V. (1988). Modern Welding Technology, Oxford \& IBH Publishing Co. Pvt.Ltd, New Delhi

[3] Houldcroft, P.T. (1990). Submerged-arc welding, Woodhead Publishing Limited

[4] Gunaraj, V.; Murugan, N. (2000). Prediction and optimization of weld bead volume for the Submerged Arc Process - Part 1, Welding Journal, 286-294

[5] Gunaraj, V.; Murugan, N. (2000). Prediction and optimization of weld bead volume for the Submerged Arc Process - Part 2, Welding Journal, 331-338 
[6] Murugan, N.; Gunaraj, V. (2005). Prediction and control of weld bead geometry and shape relationships in submerged arc welding of pipes, Journal of Materials Processing Technology, Vol.168, 478-487

[7] Taguchi, G. (1990). Introduction to quality engineering, Asian Productivity Organization, Tokyo

[8] Peace, G.S. (1993).Taguchi methods: A hand-on approach, Addison-Wesley

[9] Edwin Raja Dhas, J.; Kumanan, S. (2007). Determination of SAW process parameters using taguchi method and regression analysis, Indian Journal of Engineering and Material science, Vol.14, 103-111

[9] Deng, J. (1989). Introduction to grey system, The Journal of Grey System, Vol.1, 1-24

[10] Tosun, N. (2006). Determination of optimum parameters for multi-performance characteristics in drilling by using grey relational analysis, International Journal of Advanced Manufacturing Technology, Vol. 28, 450-455

[11] Kuo, C. F. J.; Wu, Y. S. (2006). Optimization of the film coating process for polymer blends by the grey based Taguchi method, International Journal of Advanced Manufacturing Technology, Vol.27, 525-530

[12] Saurav Datta; Asish Bandyopadhyay; Pradip Kumar Pal, (2008). Grey-based taguchi method for optimization of bead geometry in submerged arc bead-on-plate welding, International Journal of Advanced Manufacturing Technology, Vol. 39, 1136-1143

[13] Hsiao, Y. F.; Tarng, Y. S.; Huang W. J (2008). Optimization of Plasma Arc Welding Parameters by Using the Taguchi Method with the Grey Relational Analysis, Materials and Manufacturing Processes, Vol. 23, 51-58

[14] Sukhomay Pal; Santosh, K.; Malviya; Surjya, K. Pal; Arun, K. (2009). Samantaray Optimization of quality characteristics parameters in a pulsed metal inert gas welding process using grey-based Taguchi method, International Journal of Advanced Manufacturing Technology, Vol.44, 1250-1260

[15] Ahilan, C.; Kumanan, S.; Sivakumaran, N. (2010). Grey relational analysis, Application of taguchi based grey relational analysis in multi objective optimization of turning process, International Journal of Advances in Production Engineering and Management, Vol. 5, No. 3, 171-180

[16] Kamal Jangra; Ajai Jain; Sandeep Grover. (2010). Optimization of multiple machining characteristics in wire electrical discharge machining of punching die using Grey relational analysis, Journal of Scientific and Industrial Research , Vol. 69, 606-612

[17] Saurav Datta.; Asish Bandyopadhyay.; Pradip Kumar Pallnt. (2008). Solving multi-criteria optimization problem in submerged arc welding consuming a mixture of fresh flux and fused slag, Journal of Advanced Manufacturing Technology, Vol. 35, 935-942

[18] Zadeh, L. (1965). Fuzzy sets, Information Control, Vol.8, 338-353

[19] Tarng, Y. S.; Yang W. H.; Juang S. C . (2000). The Use of Fuzzy Logic in the Taguchi Method for the Optimisation of the Submerged Arc Welding Process, International Journal of Advanced Manufacturing Technology, Vol.16, 688-694

[20] Lin, J. L.; Wang, K. S.; Yan, B. H.; Tarng, Y. S. (2000). Optimization of the electrical discharge machining process based on the Taguchi method with fuzzy logics, Journal of Materials Processing Technology, Vol.102, 48-55

[21] Chiang, K.T.; Liu, N. M.; Chou, C. C. (2008). Machining parameter optimization on the die casting process of magnesium alloy using the grey-based fuzzy algorithm, International Journal of Advanced Manufacturing Technology, Vol.38, 229-237

[22] Nun-Ming Liu; Jenn-Tsong Horng; Ko-Ta Chiang. (2009). The method of grey-fuzzy logic for optimizing multi-response problems during the manufacturing process: a case study of the light guide plate printing process, International Journal of Advanced Manufacturing Technology, Vol.41,200-210

[23] Ahilan, C.; Kumanan, S.; Sivakumaran, N. (2009). Multi objective optimization of CNC turning process using grey based fuzzy logic, International Journal of Machining and Machinability of materials. Vol.5, No.4, 434-451

[24] Ankita Singh.; Saurav Datta.; Siba Sankar Mahapatra.;Tapan Singha.; Gautam Majumdar. (2011). Optimization of bead geometry of submerged arc weld using fuzzy based desirability function approach, Journal of Intelligent Manufacturing. DOI 10.1007/s10845-011-0535-3

[25] Fisher, R. A. (1925). Statistical method for research worker, Oliver \& Boyd, London

[26] Dubois, D.; Prade, H.M. (1980). Fuzzy sets and systems: theory and applications, Academic Press, New York

[27] Zimmermann. (1985). Fuzzy set theory and its applications, Kluwer, London

[28] Zadehm, L. (1995). The MathWorks User guide, Version 2, Fuzzy Logic Toolbox 